\title{
An Unusual Case of Polypoid Angiodysplasia
}

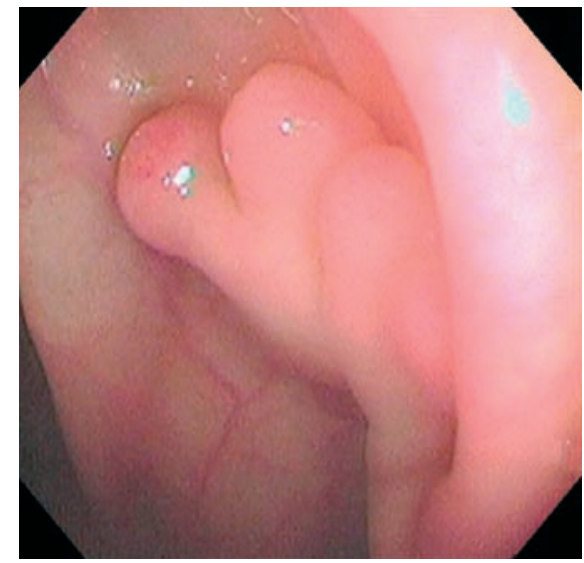

Figure 1 A healthy 59-year-old man underwent colonoscopy because of a positive fecal blood test. A yellowish-white lobulated polyp, $15-\mathrm{mm}$ in size with a "baby's hand"-like morphology, was seen. Endoscopic polypectomy for suspected leiomyoma was performed without complication.

N. Kakushima', M. Fujishiro', N. Yahagi', M. Oka', K. Kobayashi' ${ }^{1}$, T. Hashimoto',

H. Miyoshi', Y. Moriyama' ${ }^{1}$, S. Enomoto ${ }^{2}$, M. Iguchi ${ }^{2}$, M. Ichinose ${ }^{2}$, M. Omata ${ }^{1}$

${ }^{1}$ Department of Gastroenterology,

Faculty of Medicine, University of Tokyo, Tokyo, Japan

2 Second Department of Internal Medicine, Wakayama Medical College, Wakayama, Japan
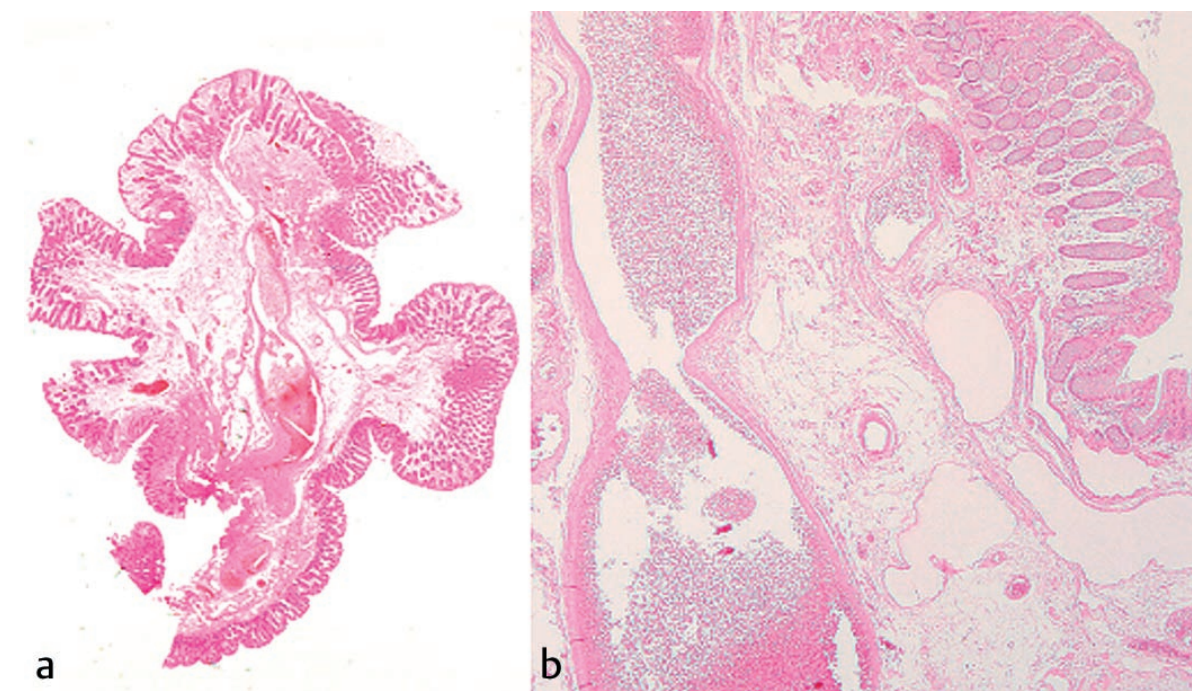

Figure 2 The edematous stroma was filled with enlarged serpiginous veins and arterioles, leading to a diagnosis of angiodysplasia. Angiodysplasia is one of the major causes of lower gastrointestinal bleeding, often encountered during emergency colonoscopy. The typical endoscopic appearance is often reported to be a slightly elevated reddish lesion, reflecting the dilated, tortuous veins in the submucosa. Lesions having a polypoid morphology are extremely rare. This case suggests us the possibility of angiodysplasia as a polypoid lesion, and the need for care when performing biopsy or endoscopic polypectomy.

Corresponding Author

\section{N. Yahagi, M.D.}

Department of Gastroenterology

Faculty of Medicine

University of Tokyo

7-3-1 Hongo

Bunkyoku

Tokyo

Japan

Fax: $\quad+81-3-5800-8806$

E-mail: Yahagi-tky@umin.ac.jp 